\title{
CIRUGÍA DE NEOIMPLANTE VESICO URETERAL POR VÍA EXTRAVESICAL; MODIFICACIONES TÉCNICAS Y CONSEJOS BASADOS EN MÁS 30 AÑOS DE EXPERIENCIA
}

\author{
Ricardo Zubieta y Pedro José López.
}

Departamento de Urología Pediátrica. Hospital Exequiel González Cortés. Clínica Alemana. Santiago. Chile.

\begin{abstract}
Resumen.- OBJETIVO: La relación alterada entre el largo y diámetro del ureter intramural en su recorrido en la vejiga es fundamental para el desarrollo de Reflujo Vesicoureteral (RVU). Por lo que el tratamiento quirúrgico debería reconstruir esta relación. Se han descrito varias técnicas quirúrgicas para lograr este objetivo. Después de más de 30 años de experiencia, en nuestras manos la técnica de neoimplante ureteral extravesical de Lich-Gregoir presenta excelentes resultados, por lo que la describimos paso a paso, haciendo hincapié en las modificaciones que se le han hecho, así como en su aplicación laparoscópica.
\end{abstract}

MÉTODOS: La técnica de neoimplante ureteral extravesical de Lich-Gregoir es descrita en su totalidad, reforzando aquellos detalles técnicos que permitirían lograr mejores resultados. Se comenta a la vez las variables de la técnica en forma laparoscópica. Se realiza también una revisión retrospectiva de las fichas clínicas y estudios imaginológicos de pacientes sometidos a neoim-
Ricardo Zubieta.

Jefe Cirugía Pediátrica

Hospital Exequiel González Cortes

Ramón Subercauseaux 1528

San Ramon. Santiago. (Chile).

bego@entelchile.net plante vesicoureteral en el periodo de 1974 - 2006 en el Hospital de Niños Dr. Exequiel González Cortes. Los criterios de inclusión fueron pacientes operados por $R V U$ primario con técnica de Lich-Gregoir, excluyendo los RVU secundario. Se registra edad, sexo, grado radiológico del RVU, bilateralidad, tiempo quirúrgico, y desarrollo de complicaciones como reflujo persistente, reflujo contralateral, infecciones urinarias postoperatorias, retensión urinaria, obstrucción postoperatoria, necesidad de reintervención, grado de insuficiencia renal, así como seguimiento a largo plazo.

RESULTADOS: En los 33 años estudiados hubo 267 pacientes con RVU primario que requirieron cirugía, 379 uréteres neo-implantados en forma extravesical, 112 (42\%) correspondian a RVU bilateral. La edad promedio al momento de la cirugía fue de 4 años 13 meses a 16 años; 156 niñas (58\%) y 111 hombres (42\%). Todos los pacientes presentaban RVU primario, y un 63\% era de alto grado (grado IV-V). Todos los uréteres fueron neo-implantados sin modelaje en un tiempo promedio de cirugía de 62 minutos para la técnica abierta. Después de un seguimiento promedio de 2 años 12 meses - 5.5 años) un $7 \%$ de los pacientes han presentado complicaciones. Nuestra tasa de reoperaciones es de 1.3\%. No se encontraron niños con retención urinaria postoperatoria. Nuestra tasa de éxito en resolver el RVU con esta técnica es $98.5 \%$.

CONCLUSIONES: Basados en los resultados los autores opinan que la técnica de neoimplante ureteral extravesical de Lich-Gregoir es segura, simple, técnicamente reproducible, eficiente y con poca morbilidad para resolver el RVU primario unilateral y bilateral.

Palabras clave: Reflujo vesico-ureteral. Técnica de Lich-Gregoir. Neoimplante extravesical. Infección urinaria. Laparoscopia. Modificaciones técnicas. 
Summary.- OBJECTIVES: The uneven relationship between length and diameter of the intramural ureter is essential for the development of vesicoureteral reflux (VUR). Surgical treatment should reconstruct that relationship. Several surgical techniques have been described to achieve this objective. After more than 30 years of experience, in our hands the Lich-Gregoir extra vesicoureteral reimplantation technique shows excellent results. We describe the technique step-by-step, emphasizing the modifications introduced, also in its laparoscopic version.

METHODS: The Lich-Gregoir extravesical ureteral reimplantation technique is completely described, reinforcing those technical details allowing the achievement of better results. We comment on the technical variations in the laparoscopic version. We also perform a retrospective review of the clinical records and imaging tests in patients who underwent vesicoureteral reimplantation in the period between 1974-2006 in the Hospital de Niños Dr Exequiel Gonzalez Cortés. Inclusion criteria: patients with primary VUR in which Lich-Gregoir extravesical ureteral reimplantation technique was performed. Secondary de VUR was excluded. We registered age, gender, radiological grade, bilateralism, surgical time, and development of complications such as persistent reflux, contralateral reflux, postoperative urinary tract infection, urinary retention, postoperative obstruction, reoperation, the degree of renal insufficiency, and long-term followup.

RESULTS: Over the 33 years of the study period there were 267 patients with primary VUR who required surgery. 379 ureters were reimplanted in an extravesical fashion, 112 (42\%) corresponded to bilateral VUR. Mean age at the time of surgery was 4 years (from three months to 16 years); 156 patients (58\%) were girls and 111 boys (42\%). All patients presented primary VUR, and $63 \%$ of them were high grade (grades $(V-V)$. All ureters were reimplanted without modelling in a mean surgical time of 62 minutes for the open technique. After a mean follow-up of two years $(2$ months- $5.5 \mathrm{yr}$.) $7 \%$ of the patients have presented complications. Our reoperation rate is $1.3 \%$. There was not any postoperative urinary retention. Our success rate for VUR resolution with this technique is $98.5 \%$.

CONCLUSIONS: Based on the results the the authors think that extravesical ureteral reimplantation following the Lich-Gregoir technique is safe, simple, technically reproducible, efficient, and with a low morbidity to resolve primary unilateral and bilateral primary VUR.

Keywords: Vesicoureteral reflux. Lich-Gregoir technique. Extravesical reimplantation. Urinary tract infection. Laparoscopic. Technical modifications.

\section{INTRODUCCIÓN}

Se define Reflujo Vésico - Ureteral (RVU) el paso de orina desde la vejiga al tracto urinario superior debido a un defecto en la unión vésico-ureteral (VU). Su incidencia se calcula entre $0.4-1.8 \%$ de la población pediátrica que no ha presentado Infección del Tracto Urinario (ITU), y cerca de un 10 - 40\% en pacientes que si la han presentado. Durante el periodo de Recién Nacido (RN), se ve más en hombres que en mujeres, situación que se invierte cerca del año de vida. El RVU se encuentra más en la población blanca. Por otro lado se encontró que un 30 - 35\% de los hermanos (as) asintomáticos presentan RVU en estudios de screening.

\section{FISIOPATOLOGÍA}

Para que exista RVU se sabe que la relación entre el largo del uréter intra-mural en su recorrido en la vejiga y el diámetro del mismo debe estar alterada (menor 4-5: 1) (Figura 1). Esto puede afectarse a nivel del proceso ureteral en la etapa embriológica $16^{\circ}$ semana de gestación), o por patologías asociadas (Síndrome de Prune Belly, duplicación ureteral, entre otros), que afecten la llegada del uréter, la calidad del músculo o la capacidad del reservorio.

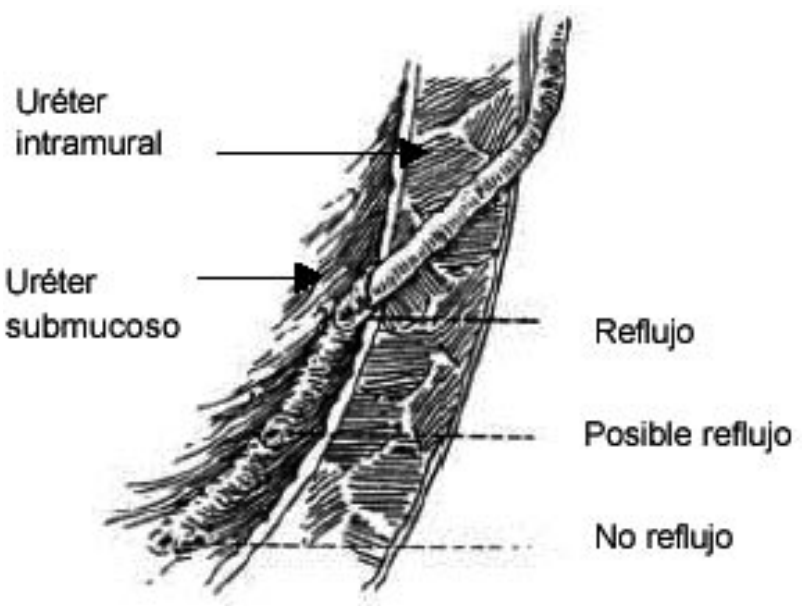

- Válvula uretero-vesical.

- Uréter intramural y submucoso.

- Posibilidades de reflujo de acuerdo a la longitud del túnel.

FIGURA 1. Unión uretero-vesical donde se aprecia la entrada en forma oblícua con un recorrido intramural largo. 
Actualmente se está estudiando si la composición fisiopatológica de la unión V-U esta alterada, lo que podría explicar o influir en la presencia de RVU. Lo cierto es que la causa real del RVU aún es incierta.

\section{CLASIFICACIÓN}

La clasificación de RVU incluye divisiones entre primario y secundarios a otra patología, simples y complejos, pero en la práctica se usa la clasificación internacional del RVU (Figura 2). Comúnmente se realiza una uretro-cistografia miccional seriada (UCG) para evidenciar RVU y poder clasificarlo, lo que orienta a pronóstico de resolución espontánea y por ende a posibles tratamientos.

\section{CLÍNICA - ESTUDIO}

El RVU no da sintomatología "per se". Lamentablemente se detecta por estar asociado a otras patologías o por sus complicaciones. Cerca de un $30-40 \%$ de los RVU se diagnóstica post estudio de una ITU. Por otro lado un $40 \%$ de los diagnósticos antenatales de hidronefrosis están asociados a RVU.

El estudio básico de RVU es con ecografía renal-vesical (ERV) y UCG. En determinados casos se pueden usar cintigramas renales para evaluar la fun-

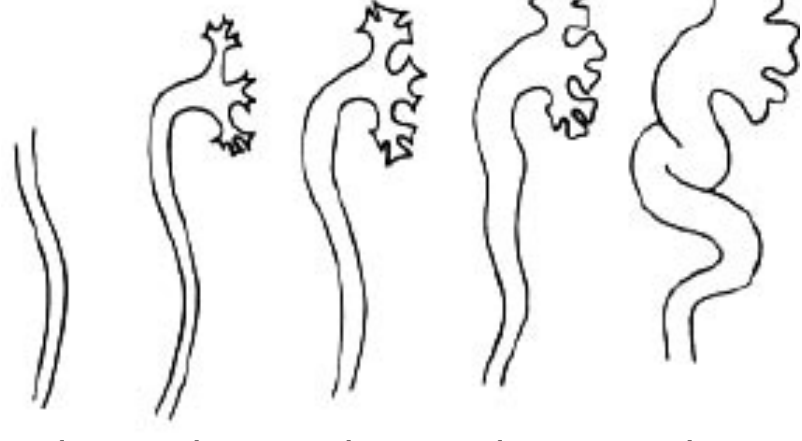

Grado 1 Grado 2 Grado 3 Grado $4 \quad$ Grado 5

\section{FIGURA 2. Clasificación internacional del RVU} Grado 1: Se llena sólo el ureter.

Grado 2: Se llena hasta la pelvis sin dilatación.

Grado 3: Existe dilatación del sistema.

Grado 4: Se aprecia dilatación importante de cálices.

Grado 5: Existe dilatación ureteral importante. ción renal, que puede ser útil en el seguimiento del RVU. Otro estudio de medicina nuclear es la técnica de isotopía indirecta que permite evidenciar RVU con menor exposición a radiación que la UCG, pero con menos resolución anatómica, por lo que se utiliza para controles.

\section{TRATAMIENTO}

Recordando la fisiopatología, el tratamiento del RVU debería ser reconstruir la relación entre uréter intra-mural y diámetro del mismo. Sin embargo, la resolución espontánea de RVU se estima cerca de un $30 \%$ por año. Este hecho depende de factores como edad de diagnóstico, grado de RVU, patologías asociadas y bilateral.

La teoría clásica del "Big Bang" de Ransely intenta explicar como el RVU podría producir daño renal. Por lo que en espera de su resolución espontánea, se inicia tratamiento con antibióticos urinarios en dosis profilácticas.

En cuanto a la corrección quirúrgica de la relación diámetro ureteral y largo de su trayecto intra-mural, podría realizarse de dos maneras; (1) aumentando el largo del trayecto con técnicas que reconstruyan el tunel con o sin reinserción del ureter, (2) disminuyendo el diámetro del orificio ureteral con técnicas de inyección de sustancias en forma endoscópica.

\section{Las indicaciones para intervenir quirúrgicamente a un niño con RVU son:}

- RVU que no se resuelve espontáneamente en el tiempo, o que por estadística será poco probable que lo haga (RVU Grado V).

- RVU que a pesar de estar con tratamiento antibiótico profiláctico presenta complicaciones como ITU.

- RVU que se acompaña de patología anatómica asociada (divertículos, ureteroceles, obstrucción unión vésico ureteral).

- RVU que se acompaña de deterioro de la función renal.

- Entorno familiar que haga poco probable que siga tratamiento antibiótico profiláctico, o en su defecto padres que no deseen estar con antibióticos por largo tiempo. 


\section{TÉCNICA QUIRÚRGICA DE NEOIMPLANTE VESICO URETERAL POR VÍA EXTRAVESICAL}

La técnica quirúrgica de neoimplante vésico ureteral por vía extravesical, comúnmente conocida como Linch-Gregoir o simplemente Gregoir fue descrita por primera vez en 1961. Su objetivo principal es mejorar la relación túnel-diámetro ureteral por medio del saneamiento del túnel.

Si bien los resultados publicados por sus autores en un inicio fueron prometedores, su expansión masiva entre los urólogos pediatras no fue del todo exitosa. Sus principales críticas fueron en relación a la retención urinaria sufrida por algunos pacientes, al seccionar parte del músculo detrusor, y por ende su inervación.

Nuestra experiencia original fue similar a la descrita en la literatura, pero una vez que fue modificada por el autor principal (RZ), los resultados fueron mejorando hasta lograr una tasa de éxito cercano al $99 \%$.

Actualmente esta técnica ha adquirido mayor aprobación entre urólogos pediatras, debido a que su abordaje extra-vesical disminuye la presencia de espasmos vesicales y las complicaciones son mínimas. La recomendación actual es realizar esta técnica como primera opción frente a un RVU unilateral.

\section{Detalles Técnicos}

- Se realiza una incisión de Pfanestiell baja, (Figura 3).

- Después una disección supra-aponeurótica hasta la inserción del ombligo, cuidando la hemostasia de los vasos perforantes.

- Con electrobisturí, se busca el punto medio entre los dos músculos rectos (línea sin contracción) y se realiza una incisión en línea media desde el ombligo hasta la sínfisis pubiana, sin sección muscular. Esto expone la vejiga, (Figura 4).

- Una vez expuesta la vejiga, se diseca el peritoneo tanto de la cara anterior como lateral, manteniendo una cirugía en espacio extra-peritoneal, (Figura 5).

- Se localiza el uréter correspondiente. Esta maniobra no siempre es tan simple, por lo que un punto anatómico de referencia importante es la arteria umbilical obliterada, donde el uréter debe pasar en forma distal o baja en forma interna a ella, (Figura 6).
- Una vez encontrado el uréter, se diseca en forma distal hasta su inserción en la cara lateral de la vejiga, (Figura 7), desplazando los elementos neurovasculares que van a la pared vesical.

- Disección proximal del peritoneo lateral extendiendo el trayecto donde se neoimplantará el ureter.

- Exponiendo la cara más lateral de la vejiga, lo que se logra con tracción suave con pinza tipo Allis al uréter, se diseña el túnel a construir, (Figura 8).

- Una vez que se diseña en túnel tanto en su orientación como en su largo, se procede a la sección del mismo. Esto se logra con electrocoagulación sobre la vejiga en forma superficial, y así se evita sangramiento muscular.

- Luego se secciona con tijeras de punta roma hasta que se logra protrusión de la mucosa vesical en todo su trayecto (Figura 9). En caso de producirse una pequeña perforación de la mucosa, esta se puede reparar con un punto de material reabsorbible (Monocry| ${ }^{\circ}$ ) sin problemas. Si la perforación es muy pequeña o se produce momentos antes de neoimplantar el uréter, no siempre es necesario repararla.

- Otra de las modificaciones que le hemos hecho a la técnica original es la disección en $Y$ a ambas caras laterales de la boca de inserción del uréter. Con este detalle se logra que el uréter repose sobre la mucosa vesical sin posibles puentes de músculo que pudieran formar una doblez obstructiva luego del cierre, (Figura 10).

- Antes de iniciar la reconstrucción del túnel se comprueba que el largo del mismo es adecuado al ancho del uréter. Si bien es cierto que este punto es importante, hemos visto que en mega-uréteres obstructivos los resultados también son buenos sin lograr la relación 4:5 es a 1 previamente recomendada (Figura 11), debido que con el tiempo el megaureter neoimplantado disminuiría de tamaño, recuperando su relación ideal de largo y diámetro.

- Luego se reconstruye el túnel muscular con puntos separados de Vicryl en un solo plano, (Figura 12).

- Es importante que al finalizar la reconstrucción se compruebe que la entrada del uréter al neo-túnel no esté obstruída, lo que se logra con la inserción de una pinza para que tenga una amplitud adecuada.

- Cuando se ha finalizado, se deja volver la vejiga a su posición anatómica natural, comprobando que la entrada del uréter este en forma recta sin angulaciones y en buena posición. 


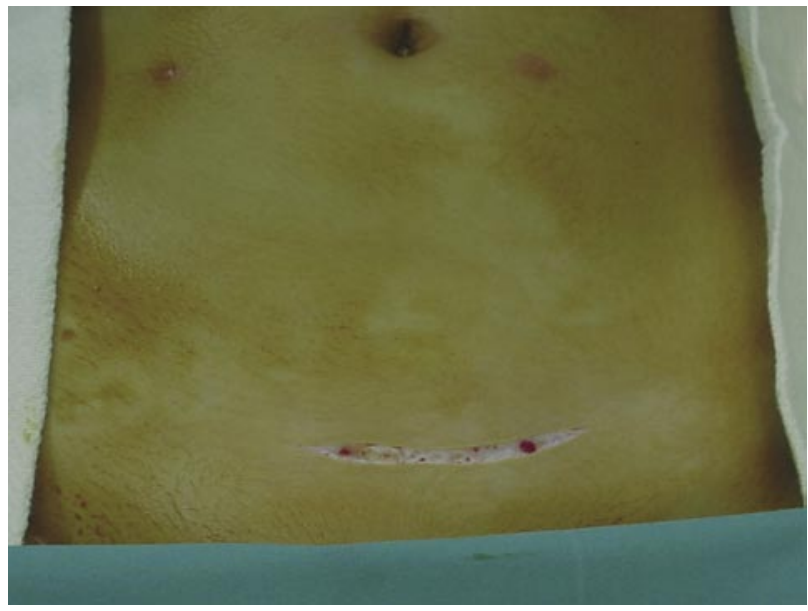

FIGURA 3. Incisión de Pfanestiell.

- En este minuto, en los niños, se coloca la cistostomía por punción (Cistofix间).

- Después de comprobar hemostasia, se comienza el cierre aponeurótico y de pared abdominal, sin necesidad de dejar drenaje de peri-vesical.

- En caso de realizar esta técnica en forma laparoscópica, los principios básicos son los mismos. Nuestra preferencia es la inserción de tres trócares; uno umbilical y uno en cada una de ambas fosos ilíacas.

- De ser necesario se puede introducir un cuarto trocar suprapúbico, o en su defecto un punto de tracción transparietal, lo que permite una mejor estabilización de la vejiga, ya sea para su disección como la reconstrucción del túnel, (Figura 13).

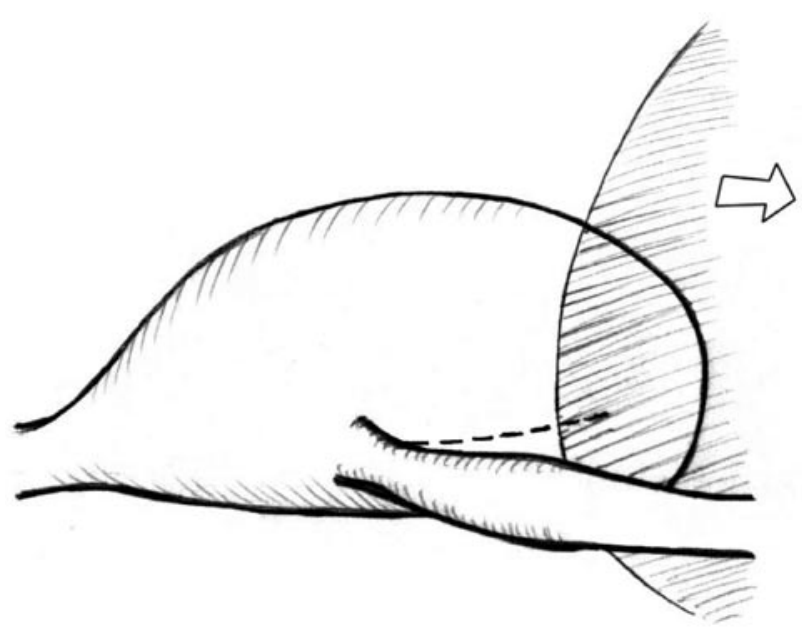

FIGURA 5. Movilización de peritoneo.

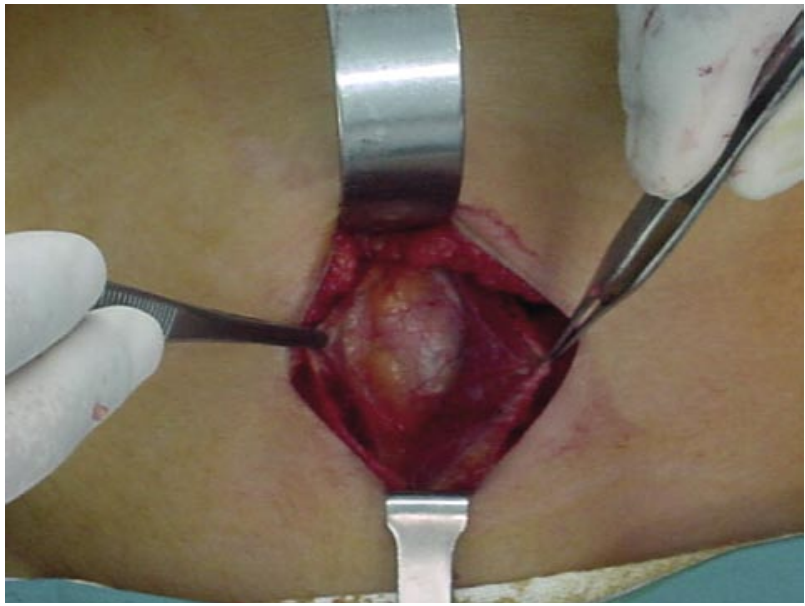

FIGURA 4. Disección supra-aponeurótico en línea media, exponiendo vejiga.

- La demarcación del túnel se realiza con electrocoagulación con Hoock, (Figura 14).

- La sutura puede ser de manera intra-corpórea o con nudos desplazados en forma extracorpórea, (Figura $15 \mathrm{~A}$ y B).

- Al finalizar, se deja drenaje vesical donde en niñas usamos sonda tipo Foley y en niños drenaje por punción supra-púbica (Cistofix ${ }^{(\times)}$). Estos drenajes quedan por 5 días, por lo que al momento del alta (24-48 horas post-cirugía) drenan a segundo pañal (en los niños menores).

- Todos los pacientes reciben analgesia oral al menos 3 días.

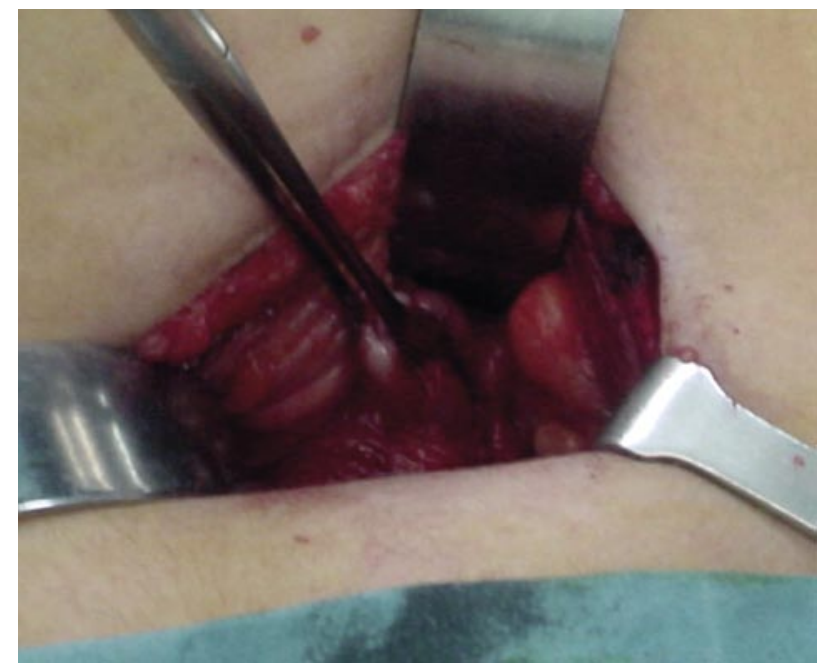

FIGURA 6. Ubicación y disección ureteral. 


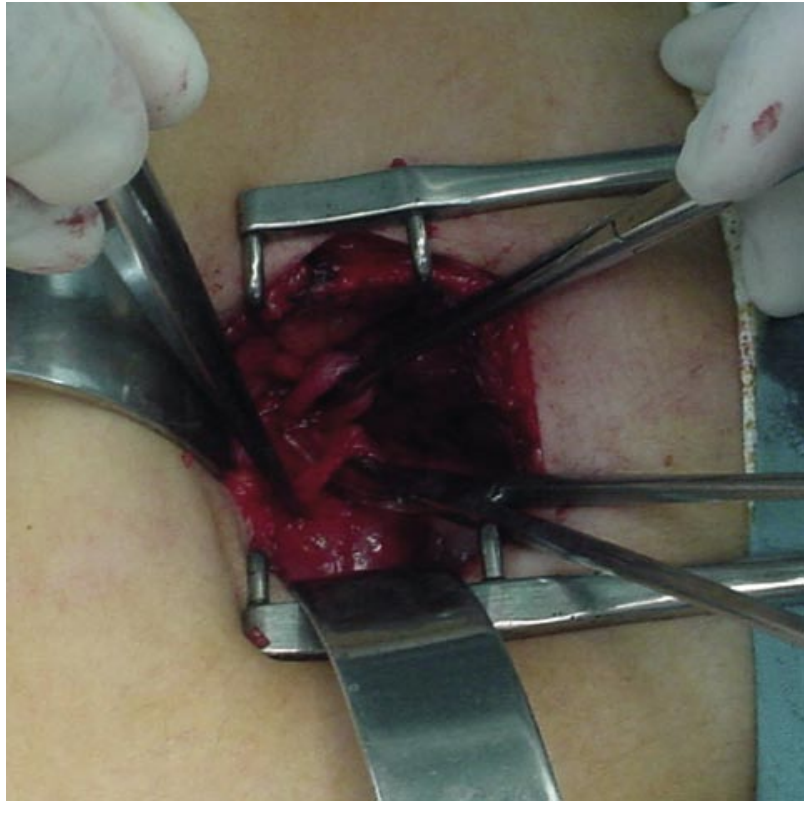

FIGURA 7. Disección de uréter hasta su entrada a la vejiga.

- Nuestra preferencia es continuar con antibióticos en dosis de tratamiento hasta el retiro de la sonda y luego en dosis de profilaxis durante las próximas 12 semanas hasta que se resuelva la inflamación o fibrosis post quirúrgica del detrusor.

- Nuestro protocolo de seguimiento con exámenes contempla un control al primer mes post-cirugía con examen de orina y ecografía renal-vesical y al año post intervención con una nueva ecografía RV y un renograma funcional (DMSA). Actualmente no realizamos nueva UCG de rutina.

\section{Resultados Post-Operatorios}

En una revisión de la utilización de esta técnica durante los últimos 33 años (1974-2006), evaluamos todos aquellos niños con RVU primario. En el periodo estudiado hubo 267 pacientes con RVU primario que requirieron cirugía, con 379 uréteres neo-implantados en forma extravesical.

La edad promedio al momento de la cirugía fue de 4 años ( 3 meses a 16 años), donde 158 eran niñas (59\%). Del grupo estudiado, 113 pacientes presentaban RVU bilateral. Todos los pacientes presentaban RVU primario, y un $66 \%$ era de alto grado (grado IV-V). Todos los uréteres fueron neoimplantados sin modelaje en un tiempo promedio de cirugía de 60 minutos.

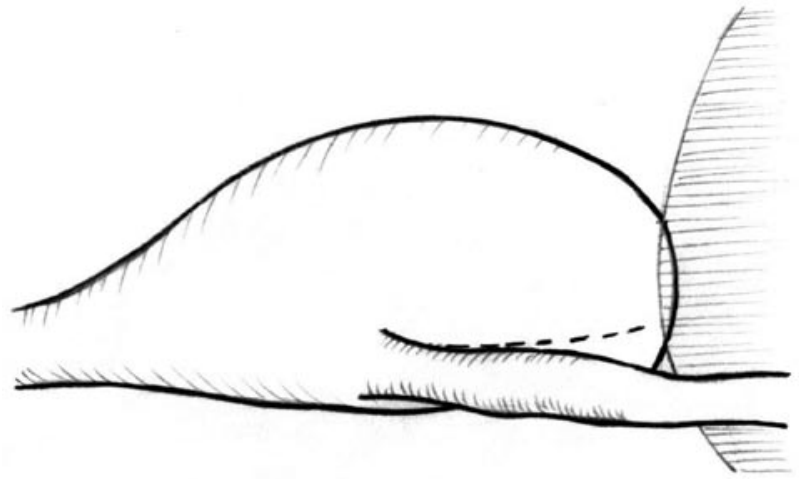

FIGURA 8. Diseño del tunel en cara lateral.

La edad promedio al momento de la cirugía fue de 4 años ( 3 meses a 16 años), donde 156 eran niñas (58\%). Del grupo estudiado 112 pacientes presentaban RVU bilateral. Todos los pacientes presentaban RVU primario, y un 63\% era de alto grado (grado (V-V). Todos los uréteres fueron neoimplantados sin modelaje en un tiempo promedio de cirugía de 60 minutos.

Después de un seguimiento promedio de 2 años (2 meses - 5.5 años), sólo 8 pacientes presenta-

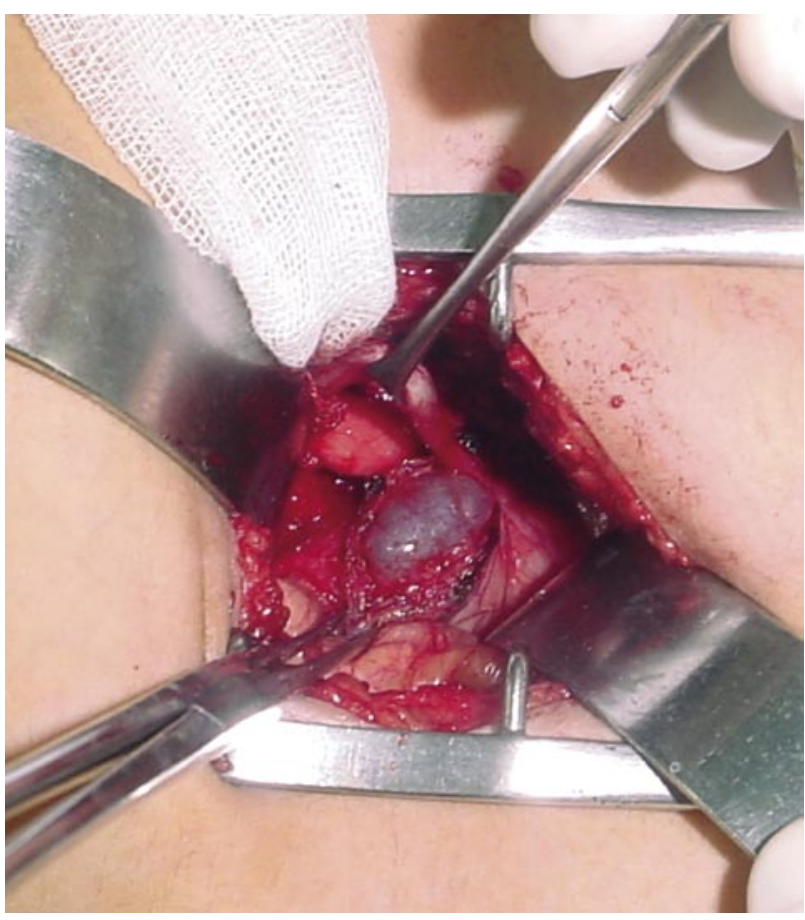

FIGURA 9. Protrusión de mucosa vesical al seccionar con tijeras. 

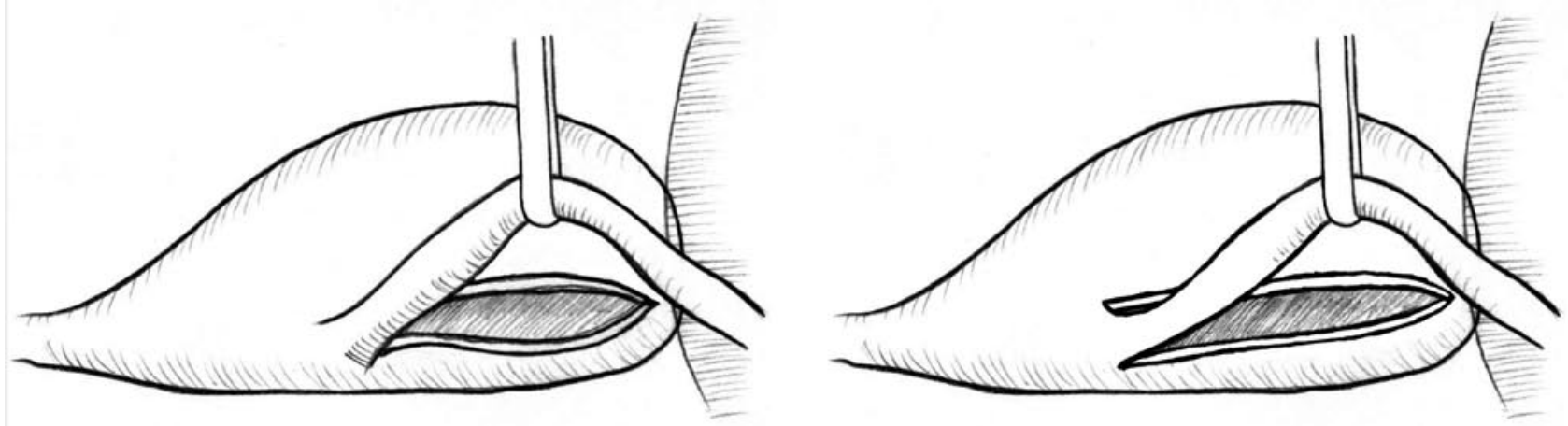

FIGURA 10. Modificación de disección en Y en caras laterales de inserción ureteral.

ron complicaciones; 4 RVU persistentes y 4 obstrucciones. Hubo otros 10 pacientes que presentaron RVU contralaterales. De los 8 pacientes que presentaron complicaciones solamente $6(1.6 \%)$ requirieron re-operación (3 obstrucciones y 3 neoimplantes por RVU persistentes). En los otros 2 pacientes, el ureter obstruido se resolvió con la instalación temporal de una sonda ureteral tipo Pig Tail. De los 10 RVU contralaterales en uno de ellos se inyectó Deflux® y el resto se resolvió en forma espontánea. Nuestra tasa de éxito en resolver el RVU con esta técnica es cercana al $98.4 \%$, encontrando sólo 6 niños que requirieron re-operación (1.6\%)

\section{CONCLUSIONES}

La técnica quirúrgica de neoimplante vésico ureteral por vía extravesical ha demostrado ser una técnica de utilidad en resolver el RVU primario con una tasa de éxito cercana al $98.5 \%$.

Debido a esta tasa de éxito, dentro de nuestro protocolo no controlamos a nuestros pacientes con UCG post operatoria, reservandola sólo para aquellos casos que presenten complicaciones como ITU, o alteraciones a la ecografía de control, hecho que ocurrió en 8 de 379 uréteres.
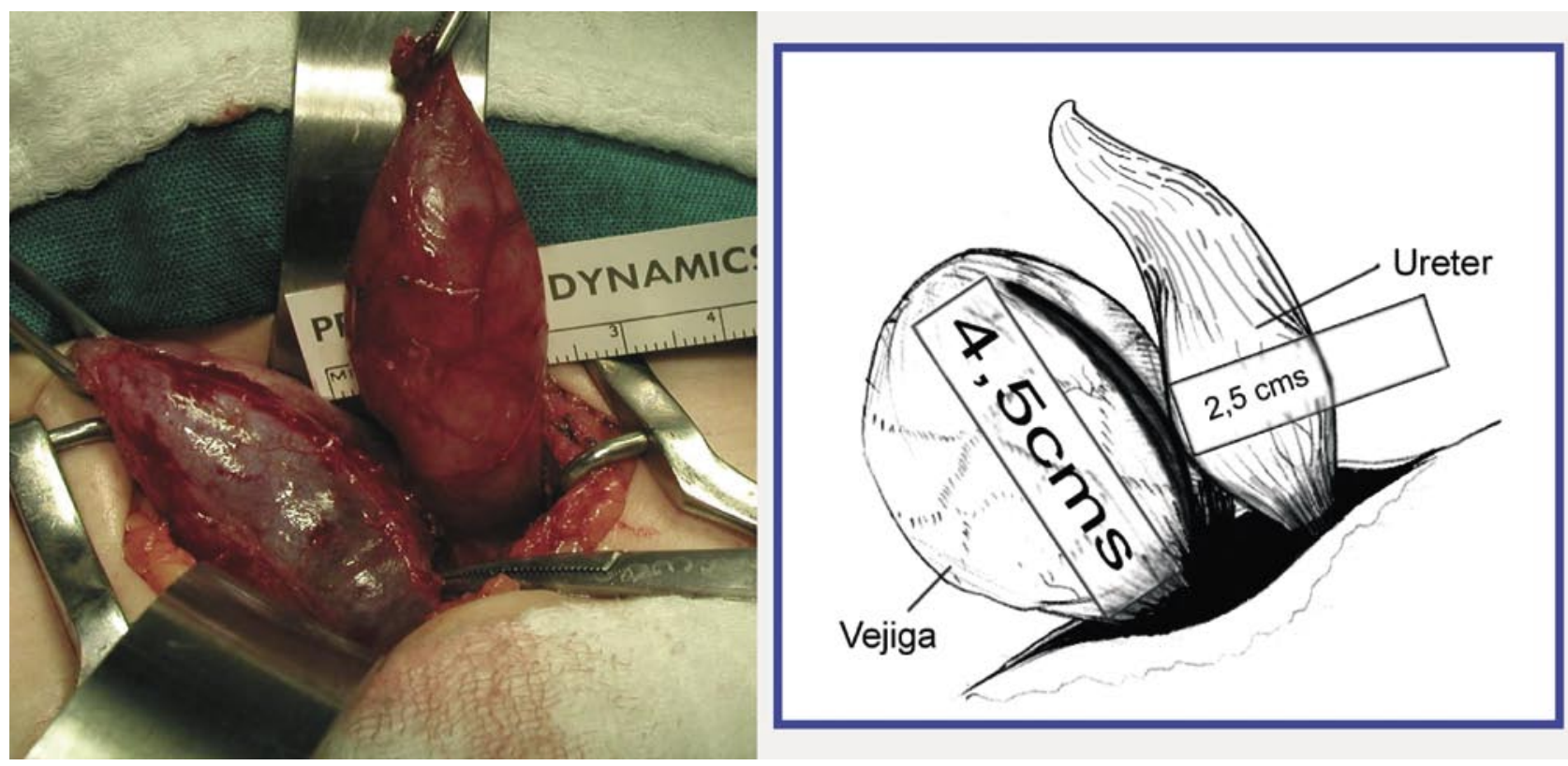

FIGURA 1 1. Reconstrucción ureteral en megaureter. 

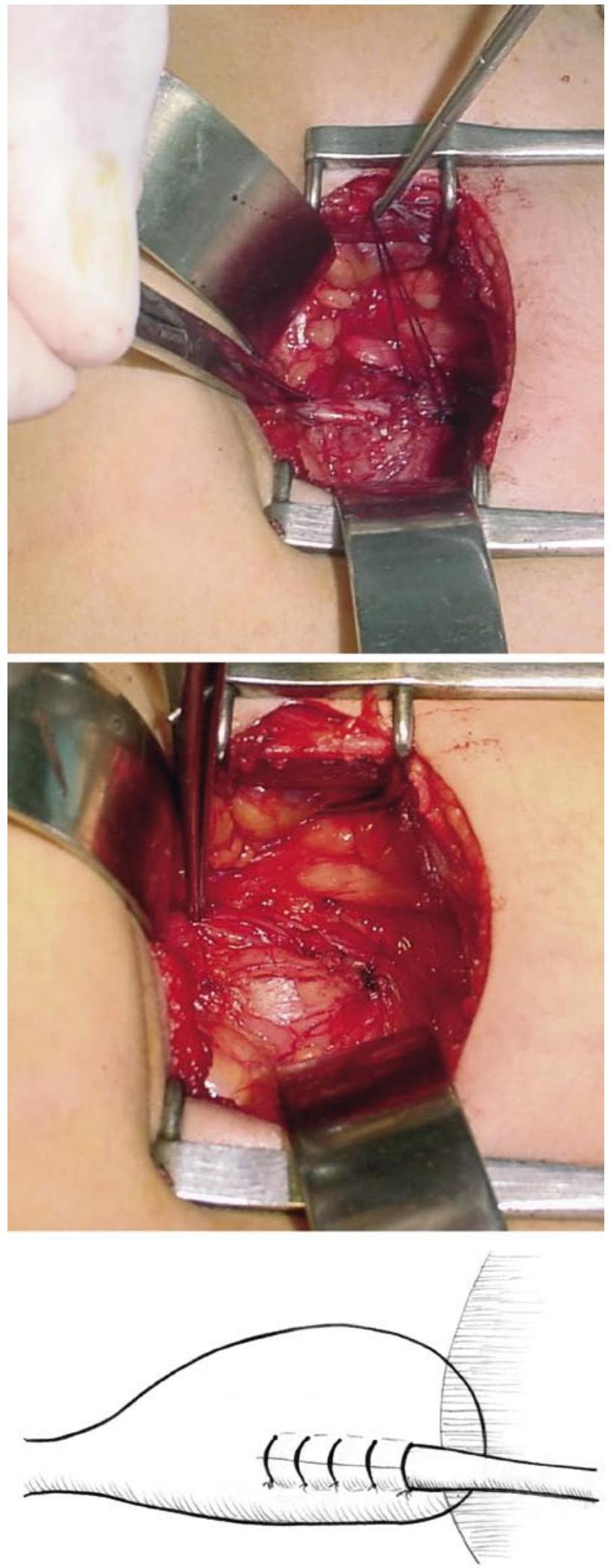

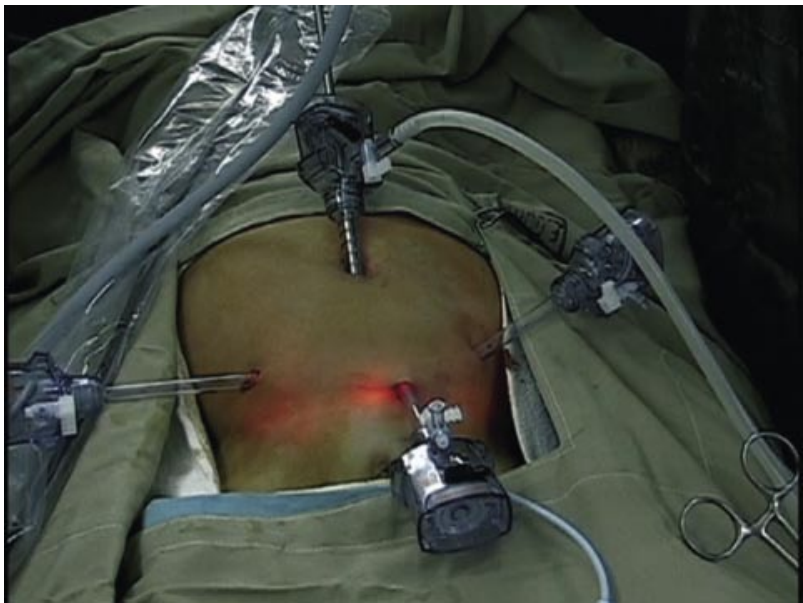

FIGURA 13. Trócares utilizados para neoimplante laparoscópico.

A la vez esta técnica ha demostrado su utilidad y validez en situaciones como megauréteres, incluso aquellos de carácter obstructivo, en neoimplante de uréteres de riñones transplantados, así como en neoimplante de ostomas urinarios cateterizables (Mitrofanoff).

A nuestro parecer los detalles de este éxito consisten en la correcta localización del uréter, en una buen diseño del sentido del túnel, en evitar dobleces en la boca del mismo, en el uso de sonda vesical, en un estudio adecuado que descarte disfunción vesical y en un protocolo de controles adecuado.

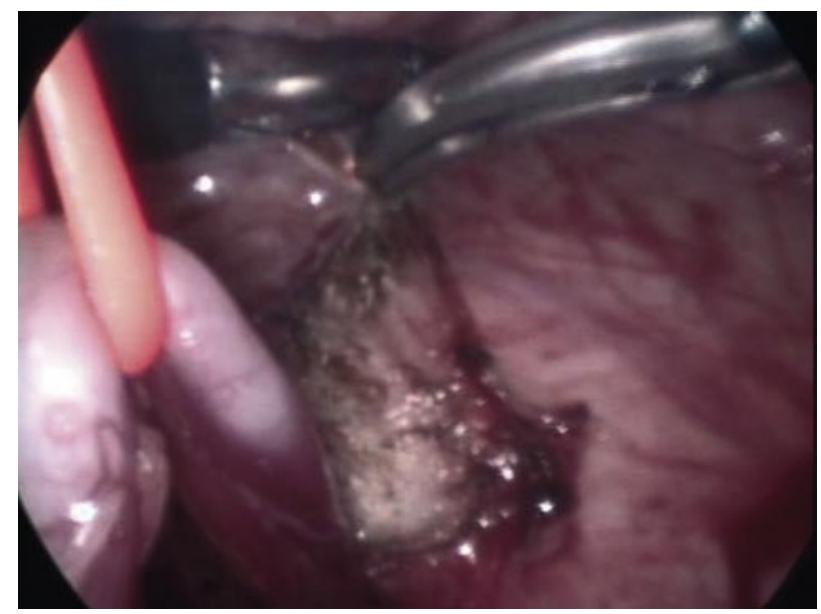

FIGURA 14. Marcación del tunel con electrocoagulación.

FIGURA 12. Reconstrucción ureteral con puntos separados. 

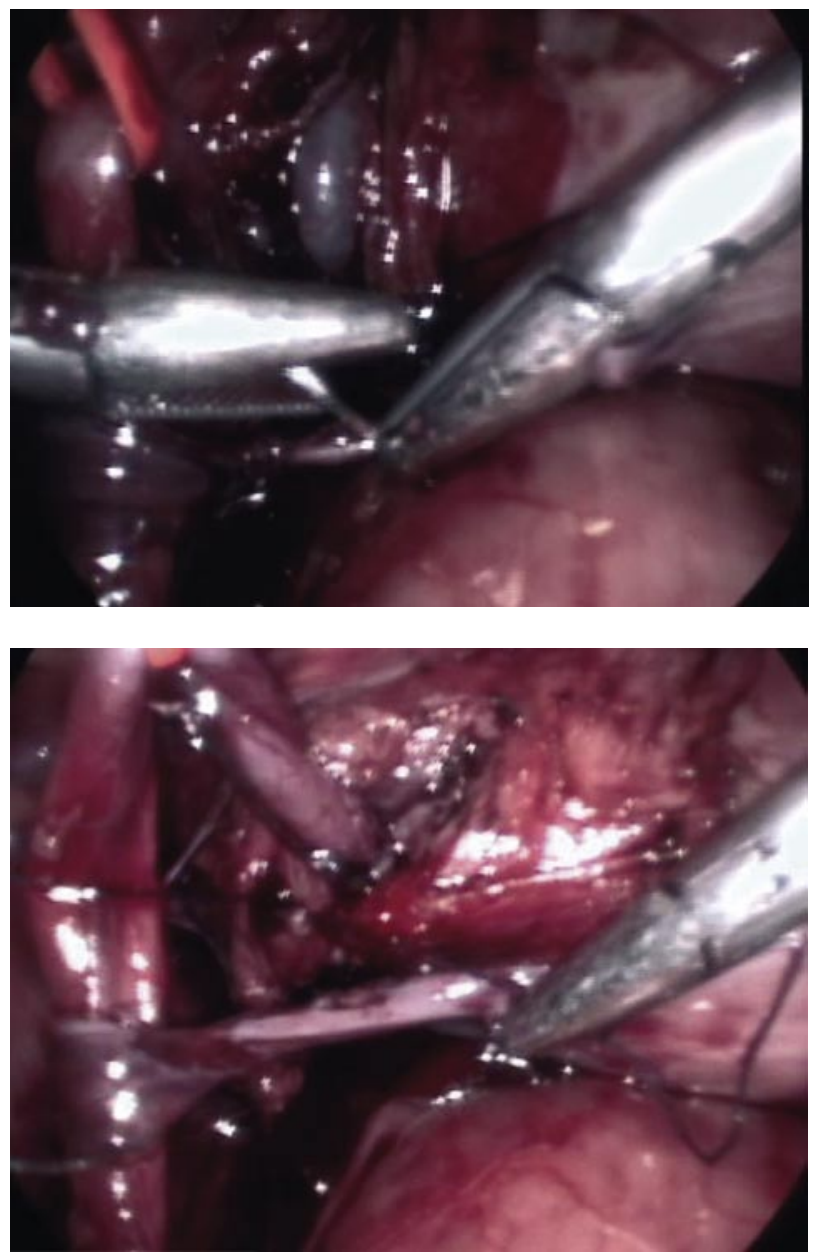

FIGURA 15. Sutura intracorpórea en neoimplante laparoscópico.

\section{BIBLIOGRAFÍA y LECTURAS RECOMENDADAS (*lectura de interés $y^{* *}$ lectura fundamental)}

1. BELMAN, B.: "Vesicoureteral reflux". Pediatric Clinics of North America, 44: 1171, 1997.

2. EWALT, D.: "Renal infection, abscess, vesicoureteral reflux, urinary litiasis, and renal trombosis". Pediatric Surgery, O`Neill, J.; Rowe, M.; Grosfeld, J.; Konkalsrud Coran, A.; Ed., Mosby, pag. 1609-1621, 1998.

3. HEIDENREICH, A.; ÖZGUR, E.; BECKER, T.: "Surgical management of vesicoureteral reflux in pediatric patients". World J. Urol., 22: 96, 2004.

4. KAEFER, M.; DIAMOND, D.: "Vesicoureteral reflux". Pediatric Urology Practice, Gonzalez, E.; Bauer, S.; Ed., Lippincott Williams \& Wilkins, pag. 463-486, 1999.

5. MOURIQUAND, P.; "Surgical treatment in vesicoureteric reflux". Pediatric Surgery, Spitz, L.; Coran, A.: Ed. Chapmann \& Hall Medical, pag. 643- 653, 1995.

6. PARROT, T.; SKANDALAKIS, J.; WOOD, S.: "The kidney and the ureter". Embriology for surgeons, Skandalakis, J.; Wood, S.; Ed. Willams \& Wilkins, pag. 594- 663, 1994.

7. PODESTA, M.: "Refluxo vesicoureteral". Cirurgia pediatrica, Maksoud, J.; Ed. Revinter, pag. 1177- 1190, 1998.

8. SHELDON, C.; MINEVICH, E.; WACKSMAN, J.: "Urinary Tract Infections and vesicoureteral reflux". Pediatric surgery, Aschcraft, K.: Ed. W.B. Saunders Company, pag. 706-724, 2000.

9. ZUBIETA, R.; LÓPEZ, P.J.; LAGOS, W. y cols.: "Neoimplante de Gregoir en megauréter obstructivo: ¿Es necesaria su remodelación?”. Rev. Chilena Urología, 70: 4, 2005. 